\title{
Apolipoprotein A1 is A Protective Factor for Male Papillary Thyroid Cancer Patients. A Cross-Sectional Study of Single Academic Center in China.
}

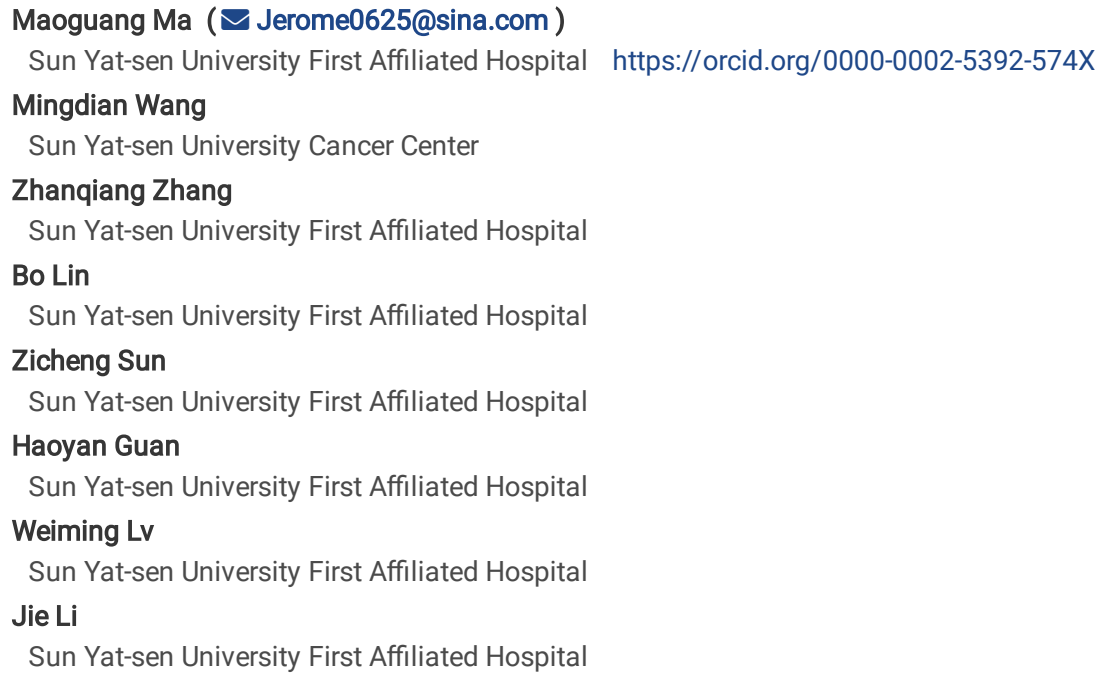

Version of Record: A version of this preprint was published at BMC Endocrine Disorders on April 14th, 2021. See the published version at https://doi.org/10.1186/s12902-021-00714-9. 


\section{Abstract}

Background: Papillary thyroid cancer (PTC) is the most common type of thyroid cancer and the incidence of PTC has continued to increase over the past decades. Many studies have shown that obesity is an independent risk factor for PTC and obese PTC patients tend to have a relative larger tumor size and higher grade of tumor stage. Obesity is associated with disordered lipid metabolism and the relationship between serum lipids and PTC remains unclear. Therefore, this study aimed to investigate the association between serum lipid level and PTC.

Methods: We retrospectively analyzed 1018 PTC patients diagnosed and treated in our hospital, all these cases were first diagnosed with PTC and had complete clinical information including ultrasound reports before surgery, serum lipid (CHOL, TG, HDL-c, LDL-c, Apo-A1, Apo-B, Apo-E) results, surgical records and pathological reports.

Results: None of these lipid markers were associated with tumor size in the whole cohort and in the female group. In the male group, on crude analysis, Apo-A1 showed a marginally significant association with tumor size, [OR=0.158 (0.021-1.777)], $p=0.072$. After adjusting for age and multifocality, Apo-A1 showed a significant association with tumor size [OR=0.126 (0.016-0.974)], $\mathrm{p}=0.047$. This association become more apparent in a young male subgroup, [OR=0.051 (0.005-0.497)], p=0.009. CHOL, TG, HDL-C, LDL-c, Apo-B, Apo-E did not show significant association with tumor size. As for LNM, neither in the male group nor in the female group were found to be associated with any serum lipid biomarkers.

Conclusion: As PTC incidences continues to increase, our finding a negative association between PTC and apoA-1 imply that these lipids are protective factors for male PTC patients and provide us new clues about PTC and lipid metabolism, which may contribute to further investigation concerning diagnosing and preventing this most common type of thyroid cancer.

\section{Background}

The incidence of PTC increased drastically in the past few decades, meanwhile, many researchers had noticed a simultaneously increased morbidity of obesity. Many studies had been launched and completed to probe the correlation between these PTC and obesity which incidence rate zoomed in the same period [1]. A pooled analysis in 2014 revealed that body mass index (BMI) and body fat percentage were significantly associated with increased risk of PTC in a population composed of Americans, Italians and Germans [2]. Furthermore, a meta-analysis comprised 12199 thyroid cancer cases reported that a statistically significant greater risk of thyroid cancer (including papillary thyroid cancer, follicular thyroid cancer and anaplastic thyroid cancer) was present in overweight and obese individuals [3]. Besides higher risk of morbidity of PTC, obesity was associated with larger tumor size and marginally significantly associated with advanced tumor stage according to a population-based study from Nevada [4]. Serum lipids are closely related with obesity and BMI and abnormal lipid metabolism is a common feature in many cancers, such as breast cancer and clear-cell renal carcinoma $[5,6]$. While the correlation between serum lipid and PTC remains elusive.

The aim of this study is to elucidate the relationship between serum lipids and extent of PTC at diagnosis, through the use of a population-based samples. This study explores whether routinely measured serum lipids are associated with tumor size, multiplicity and lymph node metastasis (LNM) of PTC in a Chinese population.

\section{Materials And Methods}

\section{Study participants and data collection}

Patients newly diagnosed with papillary thyroid cancer between January 2018 and November 2019 were retrospectively analyzed in this study. The inclusion criteria were as follows: (1) primary PTC verified by pathology; (2) age 18 years old; (3) without hypolipemic agents history; (4) did not merge with other kind of diseases; (5) complete Clinical and pathological data.

The main clinical data include serum lipid level when diagnosed with PTC, age, gender, ultrasound evaluation before surgery and pathological reports after surgery.

Serum lipid markers include CHOL, TG, HDL-c, LDL-c, Apo-A1, Apo-B, Apo-E. Age was classified as $₫ 55$ versus 55 and older. Gender was male or female. Tumor size was classified as $\leq 2 \mathrm{~cm}$ versus $₫ 2 \mathrm{~cm}$. Information about lymph node dissection was retrieved from surgical records and pathological reports, 5 or more lymph nodes presented in pathological reports were considered lymph node dissection had been performed in the previous surgery. Cancer stage was determined through the American Joint Committee on Cancer (AJCC) TNM Staging For Thyroid-Differentiated and Anaplastic Carcinoma ( ${ }^{\text {th }}$ ed., 2017). The number of tumors was determined by ultrasound reports and pathological reports, cases with only one tumor were deemed as unifocal and with 2 or more tumors were considered as multifocal. We compared the ultrasound reports before surgery and pathological reports after surgery to find out the correct and false prediction rate of lymph node metastasis by ultrasound before surgery. Clinical and pathological data were collected from the database established by The First Affiliated Hospital of Sun Yat-Sen University. Data collection was performed by two independent researchers.

\section{Statistical analysis.}

SPSS version 23.0 was used to conduct all statistical analyses. Univariate and multivariate logistic regression models were applied to assess the influence of serum lipid level on clinical characteristics by calculating the odds ratios and their corresponding $95 \%$ confidence intervals (Cls). P value< 0.05 was considered to be statistically significant.

\section{Results}


A total of 1018 PTC patients were included in this study (Fig. 1), necessary clinicopathological information are shown in (Table 1, additional file). Among the 1018 PTC patients, 892 were under the age of 55 (87.6\%), 126 (12.4\%) were 55 years old or older. The ratio of women to men was $2.8: 1$. The tumor size of 905 (88.9\%) cases were $2 \mathrm{~cm}$ or smaller, $113(11.1 \%)$ were larger than $2 \mathrm{~cm}$. 606 (59.5\%) PTC patients were performed lymph node dissection during surgery, 398 (39.1\%) cases with lymph node metastasis verified by pathological reports. As to the TNM stage, the majority of patients had stage $\nabla$ cancers ( $96.4 \%)$, 35 and 2 patients had a stage $\nabla$ and stage $\nabla$ cancers ( $3.4 \%$ and $0.2 \%$ respectively), no patient included in this study had a stage $\nabla$ cancer. $713(70 \%)$ patients had only one tumor, $305(30 \%)$ cases had two or more tumors. 262 patients were suggested with LNM and 756 patients were regarded without LNM by the ultrasound report before surgery. Among these 756 patients, 374 (49.5\%) were performed prophylactic central lymph node dissection and 178 (23.5\%) were shown with LNM actually based on the pathological reports. The inaccurately predicted rate may be higher because there were 382 patients did not go through prophylactic lymph node dissection during the surgery.

Logistic regression univariate analysis and multivariate analysis were used to analyze the association between each of the 7 serum lipid biomarkers and tumor size or LNM or multifocality or false negative prediction of ultrasound. No statistically significant association was found in terms of this analysis (Table 2, additional file). After adjusting for age, we found that patients with high level of serum Apo-A1 were shown to have marginally significant higher odds of small tumor size relative to patients with lower level of serum Apo-A1, OR and 95\% $\mathrm{Cl} 0.514$ (0.204-1.292).

Then we divided these patients into two groups by gender ( 265 men and 753 women) and analyzed the association between each of the 8 serum lipid biomarkers with tumor size or LNM respectively. As shown in (Table 3, additional file), in the male group, on crude analysis, Apo-A1 showed a marginally significant association with tumor size OR and 95\% $\mathrm{Cl} 0.158(0.021-1.777), \mathrm{p}=0.072$. After adjusting for age and multifocality, Apo-A1 showed a significant association with tumor size OR and $95 \% \mathrm{Cl} 0.126(0.016-0.974) \mathrm{p}=0.047$.

This association become stronger in a young male subgroup ( $<55$ years old, $n=237$ ). Univariate analysis showed that Apo-A1 significantly negatively correlated with tumor size in PTC patients, OR and $95 \% \mathrm{Cl} 0.047(0.005-0.485), \mathrm{p}=0.01$. After adjusted for multifocality, a similar association was seen, OR and $95 \% \mathrm{Cl} 0.051$ (0.005-0.497), $\mathrm{p}=0.01$ (Table 4).

As for LNM, neither in the male group nor in the female group were found to be associated with any serum lipid biomarkers (Table 5, additional file).

\section{Discussion}

PTC is more common in females than in males, many studies have demonstrated that the ratio of female to man in PTC is about $3: 1$, which is consistent with our results. Moreover, male patients often showed a higher PTC mortality than the females. Sheng-Hwu Hsieh once reported that male gender was an independent risk factor for cancer recurrence and mortality in PTC. But the reasons behind this prognostic difference between genders were unknown.

Serum lipid profile has been shown to be a potential diagnostic biomarker for many cancers, such as head and neck squamous cell carcinoma, colorectal cancer and lung cancer [7-9]. The aberrant lipid biosynthesis was also showed to be associated with cancer cell migration, invasion and induction of tumor angiogenesis [10]. In addition, many studies have demonstrated that obesity is strongly related to lipid disturbances and abnormal metabolism [11, 12]. Furthermore, obesity has been regarded as a risk factor for many cancers, including thyroid cancer [1, 13], so it is natural to speculate the relationship between blood lipid and papillary thyroid cancer.

In this study, we found that patients with lower levels of serum Apo-A1 are more likely to be diagnosed with larger tumor sizes of PTC in a male cohort, especially in a young male subgroup (<55 years old), this correlation was not seen when it comes to the female cohort. Tumor size has been demonstrated as an independent predictor of recurrence in PTC in previous study (tumors $>2 \mathrm{~cm}$ associated with higher risk of recurrence than those $\leq 2 \mathrm{~cm}$ ), [14] which indicates Apo-A1 is an protective biomarkers for male PTC patients.

Apo-A1 has been proved to be associated with many cancer, furthermore, it could be used as a potential biomarker for detection and diagnosis for many cancers such as bladder cancer $[15,16]$. In a recent study, researchers observed that lower serum levels of Apo-A1 in thyroid cancer patients compared to healthy controls, indicating that Apo-A1 may play an anti-tumor role in thyroid cancer [17]. Interestingly, Apo-A1 was showed association with tumor size only in the male group, but not in the female group. This phenomenon implies that sexual hormone may influence lipid metabolism and further affect PTC tumor growth. A study from Salford once reported that low-dose testosterone administration to women for two years would result in atherogenic effects on some parameters of lipid and lipoprotein metabolism, which include HDL-C, Apo-A1 and VLDL-C [18].

Though several studies had demonstrated that Apo-A1 can be a used as a prognostic parameter in many cancers, but the mechanism of the association between high serum Apo-A1 levels and favorable prognosis in several cancers are still unknown. There are increasing evidence manifested that systemic inflammation plays an important role in contributing the development and progression of malignancies [19]. Systemic inflammatory markers, such as CRP (Creactive protein), was shown to be an independent predictor of poor outcome in patients suffered from various cancers [20-22]. A recent research unveiled that serum Apo-A1 levels showed strong negative correlation with systemic inflammatory markers including serum CRP and interleukin (IL)-8 levels and blood neutrophil count in 144 colorectal cancer patients [23], which indicate systemic inflammation may influence tumorigenesis and regulate lipid metabolism in the same period, thus, enabling some kinds of serum lipid markers to correlate with tumor characteristics and provide prognostic information.

Larger tumor size always associated with a higher risk of LNM in PTC. Although we find that lower Apo-A1 levels was significantly associated with larger tumor size in male PTC patients, but they do not show a correlation with LNM. How to precisely predict LNM before surgery is always a trouble for all surgeons. We wished to excavate some information about the relationship between lipid metabolism and LNM, but the results disappointed us in this respect.

Moreover, we found that the rate of accuracy of evaluating LNM for PTC before surgery was not satisfied, even ultrasound is the best way to predict LNM of PTC currently, the false prediction rate for TNM is about $23.5 \%$ or higher according to our analysis. More precise instruments and forecasting models for 
predicting LNM before surgery should be exploited for clinical use in the future.

\section{Conclusion}

In conclusion, the present study identified Apo-A1 is a protective factors for male PTC patients, patients with higher level of Apo-A1 are more likely to have a smaller tumor size. Gender differences exhibited in the association between PTC and serum lipid level providing us new clues to explore the origination of this cancer and the underlying molecular mechanism of lipid metabolism in PTC patients require further investigation.

\section{Abbreviations}

PTC= Papillary Thyroid Cancer; $\mathrm{CHOL}=$ Cholesterol; TG=Triglyceride; HDL-C= High-density Lipoprotein Cholesterol; LDL-C= Low-density Lipoprotein Cholesterol, Apo-A1= Apolipoprotein A1; Apo-B= Apolipoprotein B; Apo-E= Apolipoprotein E; BMI= Body Mass Index; LNM= Lymph Nodes Metastasis; AJCC= American Joint Committee on Cancer; OR= Odds Ratio; $\mathrm{Cl}=$ Confidence Interval.

\section{Declarations}

\section{Acknowledgments}

None.

\section{Authors' contributions}

MM, MW collected and analyzed the data, and draft the manuscript; ZZ, BL, ZS and HG designed and supervised the study; JL and WL revised the manuscript for important intellectual content; all authors have read and approved the final version to be published.

\section{Funding}

This study was funded by the Foundation of Guangdong Science and Technology Department (2017A010105029).

\section{Availability of data and materials}

The datasets used and analyzed in this study are available from the corresponding author on reasonable request.

\section{Ethics approval and consent to participate}

The study was conducted in accordance with The Code of Ethics of the Declaration of Helsinki and approved by the ethics committee of The First Affiliated Hospital of Sun Yat-Sen University. Informed consent was obtained from all individual participants included in the study.

\section{Consent for publication}

All the authors listed have read through the manuscript and approved for the submission. All authors have contributed to and agreed on the content of the manuscript.

\section{Competing interests}

The authors declare that they have no competing interests.

\section{References}

1. Pappa T, Alevizaki M: Obesity and thyroid cancer. a clinical update. Thyroid 2014, 24(2):190-199.

2. Xu L, Port M, Landi S, Gemignani F, Cipollini M, Elisei R, Goudeva L, Muller JA, Nerlich K, Pellegrini G et al: Obesity and the risk of papillary thyroid cancer: a pooled analysis of three case-control studies. Thyroid 2014, 24(6):966-974.

3. Schmid D, Ricci C, Behrens G, Leitzmann MF: Adiposity and risk of thyroid cancer. a systematic review and meta-analysis. Obes Rev 2015, 16(12):10421054.

4. Dieringer P, Klass EM, Caine B, Smith-Gagen J: Associations between body mass and papillary thyroid cancer stage and tumor size: a population-based study. J Cancer Res Clin Oncol 2015, 141(1):93-98.

5. Yang L, Cui X, Zhang N, Li M, Bai Y, Han X, Shi Y, Liu H: Comprehensive lipid profiling of plasma in patients with benign breast tumor and breast cancer reveals novel biomarkers. Anal Bioanal Chem 2015, 407(17):5065-5077.

6. Drabkin HA, Gemmill RM: Cholesterol and the development of clear-cell renal carcinoma. Curr Opin Pharmaco/ 2012, 12(6):742-750.

7. Li G, Da M, Zhang W, Wu H, Ye J, Chen J, Ma L, Gu N, Wu Y, Song X: Alteration of serum lipid profile and its prognostic value in head and neck squamous cell carcinoma. J Oral Pathol Med 2016, 45(3):167-172.

8. Zhang X, Zhao XW, Liu DB, Han CZ, Du LL, Jing JX, Wang Y: Lipid levels in serum and cancerous tissues of colorectal cancer patients. World J Gastroenterol 2014, 20(26):8646-8652. 
9. Ujiie H, Ding L, Fan R, Kato T, Lee D, Fujino K, Kinoshita T, Lee CY, Waddell TK, Keshavjee S et al: Porphyrin-High-Density Lipoprotein: A Novel Photosensitizing Nanoparticle for Lung Cancer Therapy. Ann Thorac Surg 2019, 107(2):369-377.

10. Baenke F, Peck B, Miess H, Schulze A: Hooked on fat: the role of lipid synthesis in cancer metabolism and tumour development. Disease Models \& Mechanisms 2013, 6(6):1353-1363.

11. Stepien A, Stepien M, Wlazel RN, Paradowski M, Banach M, Rysz J: Assessment of the relationship between lipid parameters and obesity indices in nondiabetic obese patients: a preliminary report. Med Sci Monit 2014, 20:2683-2688.

12. Sahebkar A, Simental-Mendia LE, Reiner Z, Kovanen PT, Simental-Mendia M, Bianconi V, Pirro M: Effect of orlistat on plasma lipids and body weight: A systematic review and meta-analysis of 33 randomized controlled trials. Pharmacol Res 2017, 122:53-65.

13. Iyengar NM, Gucalp A, Dannenberg AJ, Hudis CA: Obesity and Cancer Mechanisms: Tumor Microenvironment and Inflammation. J Clin Oncol 2016, 34(35):4270-4276.

14. Tran B, Roshan D, Abraham E, Wang L, Garibotto N, Wykes J, Campbell P, Ebrahimi A: The Prognostic Impact of Tumor Size in Papillary Thyroid Carcinoma is Modified by Age. Thyroid 2018, 28(8):991-996.

15. Li C, Li H, Zhang T, Li J, Liu L, Chang J: Discovery of Apo-A1 as a potential bladder cancer biomarker by urine proteomics and analysis. Biochem Biophys Res Commun 2014, 446(4):1047-1052.

16. Yao X, Gordon EM, Figueroa DM, Barochia AV, Levine SJ: Emerging Roles of Apolipoprotein E and Apolipoprotein A-I in the Pathogenesis and Treatment of Lung Disease. Am J Respir Cell Mol Biol 2016, 55(2):159-169.

17. Li D, Zhou L, Ma C, Chen W, Zhang Y, Yu S, Wang D, Zou Y, Wu J, Qiu L: Comparative analysis of the serum proteome profiles of thyroid cancer: An initial focus on the lipid profile. Oncol Lett 2019, 18(3):3349-3357.

18. Buckler HM, McElhone K, Durrington PN, Mackness MI, Ludlam CA, Wu FCW: The effects of low-dose testosterone treatment on lipid metabolism, clotting factors and ultrasonographic ovarian morphology in women. Clinical Endocrinology 1998, 49(2):173-178.

19. Diakos Cl, Charles KA, McMillan DC, Clarke SJ: Cancer-related inflammation and treatment effectiveness. Lancet Oncol 2014, 15(11):e493-503.

20. Kim WR, Han YD, Min BS: C-Reactive Protein Level Predicts Survival Outcomes in Rectal Cancer Patients Undergoing Total Mesorectal Excision After Preoperative Chemoradiation Therapy. Ann Surg Oncol 2018, 25(13):3898-3905.

21. Huang J, Baum Y, Alemozaffar M, Ogan K, Harris W, Kucuk O, Master VA: C-reactive protein in urologic cancers. Mol Aspects Med 2015, 45:28-36.

22. Szkandera J, Stotz M, Absenger G, Stojakovic T, Samonigg H, Kornprat P, Schaberl-Moser R, Alzoughbi W, Lackner C, Ress AL et al: Validation of C-reactive protein levels as a prognostic indicator for survival in a large cohort of pancreatic cancer patients. Br J Cancer 2014, 110(1):183-188.

23. Sirnio P, Vayrynen JP, Klintrup K, Makela J, Makinen MJ, Karttunen TJ, Tuomisto A: Decreased serum apolipoprotein A1 levels are associated with poor survival and systemic inflammatory response in colorectal cancer. Sci Rep 2017, 7(1):5374.

\section{Tables}


Table 1 Clnical and pathological features of patients enrolled in this study. Clinical and
pathological
features

\section{Number(\%)}

CHOL(mean)

(3.1-5.7

$\mathrm{mmol} / \mathrm{L}$ )
TG(mean)

(0.33-

$\mathrm{mmol} / \mathrm{L}$
HDL-

c(mean)

(1.09-

1.63 $\mathrm{mmol} / \mathrm{L}$ )
LDLc(mean)

$(<3.4$ $\mathrm{mmol} / \mathrm{L})$

\section{Age}

$\square 55$

$\geq 55$

$892 \square 87.6 \% \square$

$126 \square 12.4 \square$

4.7916

5.4151

$p<0.001^{*}$

1.2175

1.7145

$p=0.001^{*}$

1.2399

1.2667

$p=0.354$

3.0005

3.4528

\section{Gender}

Female

Male

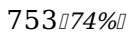

$265 \square 26 \% \square$

5.0279

4.8127

$p=0.003 *$

1.5567

$1.1813 p<0.001^{*}$

1.1051

3.2509

$1.2918 p<0.001^{*}$

2.9881

\section{Tumor size}

$\leq 2 \mathrm{~cm}$

$\square 2 \mathrm{~cm}$

Lymph node

dissection

Yes

with $L N M$

central LNM

lateral LNM

without lymph node metastasis

NO

$412 \square 40.4 \% \square$

4.8772

$p=0.822$

$p=0.413$

1.2443

1.234

$p=0.733$

3.0619

$1.3497 \quad p=0.388$

1.2658

1.2498

3.0472

398

$\underline{129}$

208

\section{TNM Stage}

In situ/localized (and ए)

Regional/distant ( $\square$ and $\mathrm{a})$

$1016 \square 99.8 \% \square$

48,688

1.279

1.2432

3.0565

$2 \quad 00.2 \%$

5.55

1.09

1.32

3.595

\section{Unifocal/Multifocal}

Unifocal
Multifocal

$713 \square 70 \% \square$

4.8734

1.2821

1.2456

3.0601

$4.858 \quad p=0.837$

1.2718

$p=0.870$

Ultrasound evaluation before surgery

\section{Suspection of LNM \\ number of correct prediction \\ number of false prediction}

uncertain

No suspection of LNM

number of correct
$262 \square 25.7 \% \square$

$231 \square 88.2 \% \square$

$15 \square 5.7 \% \square$

16ロ6.1\%凸
4.8134

$p=0.309$ 
prediction

number of false

prediction

$178 \square 23.5 \%$

uncertain

$382050.5 \%$

Table 2 Odds ratios (OR) (with 95\% CI) of levels of 7 serum lipid markers by 4 clinical characteristics.

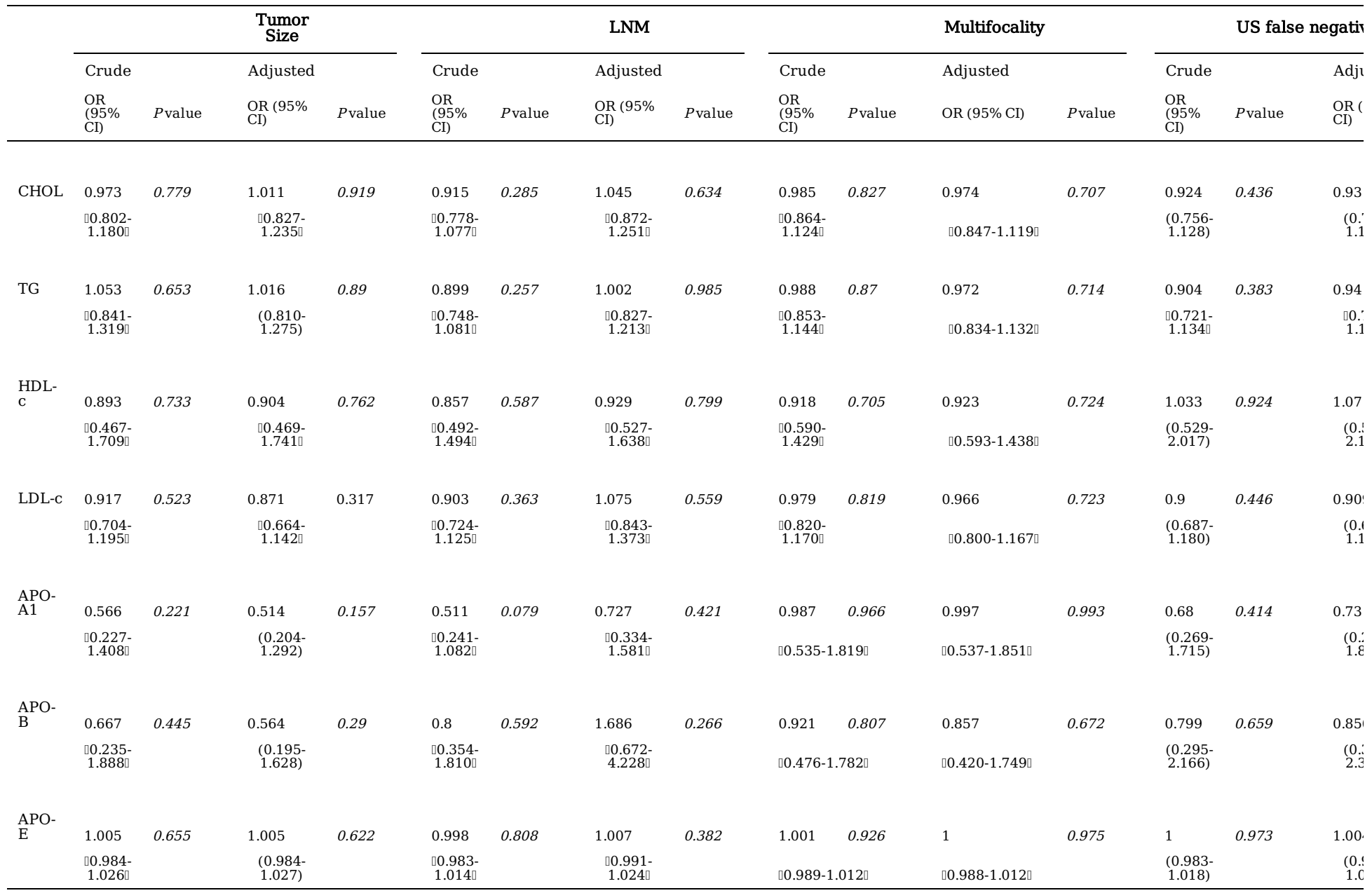


Table 3. Odds ratios (OR) (with 95\% CI) of levels of 7 serum lipid by tumor size in male and female group respectively.

\begin{tabular}{|c|c|c|c|c|c|c|c|c|}
\hline \multicolumn{5}{|c|}{ Male } & \multicolumn{3}{|c|}{ Female } & \multirow[b]{3}{*}{$P$ value } \\
\hline & Crude & & Adjusted & & Crude & & Adjusted & \\
\hline & OR (95\% CI) & $P$ value & OR $(95 \% \mathrm{CI})$ & $P$ value & OR (95\% CI) & $P$ value & OR $(95 \% \mathrm{CI})$ & \\
\hline \multirow[t]{2}{*}{ CHOL } & 0.918 & 0.644 & 0.902 & 0.577 & 0.998 & 0.984 & 0.962 & 0.749 \\
\hline & [0.637-1.321ם & & ๘0.629-1.295๘ & & [0.792-1.257】 & & $\llbracket 0.757-1.222 \square$ & \\
\hline \multirow[t]{2}{*}{ TG } & 1.008 & 0.971 & 1.046 & 0.841 & 1.105 & 0.324 & 1.075 & 0.489 \\
\hline & $\square 0.652-1.559 \square$ & & ๑0.671-1.631₫ & & $\square 0.906-1.346 \square$ & & {$[0.873-1.324 \square$} & \\
\hline \multirow[t]{2}{*}{ HDL-c } & 0.514 & 0.393 & 0.421 & 0.274 & 1.014 & 0.972 & 1.024 & 0.953 \\
\hline & $\square 0.112-2.365 \square$ & & $\llbracket 0.090-1.980 \square$ & & $\square 0.474-2.167 \square$ & & $\llbracket 0.470-2.231 \square$ & \\
\hline \multirow[t]{2}{*}{ LDL-C } & 0.79 & 0.372 & 0.788 & 0.358 & 0.974 & 0.87 & 0.921 & 0.626 \\
\hline & ¿0.471-1.325凸 & & $\llbracket 0.473-1.310 \square$ & & ¿0.709-1.337〕 & & $\llbracket 0.662-1.282 \square$ & \\
\hline \multirow[t]{2}{*}{ APO-A1 } & 0.158 & 0.072 & 0.126 & 0.047 & 0.79 & 0.671 & 0.744 & 0.598 \\
\hline & [0.021-1.177₫ & & ๑0.016-0.974₫ & & $\square 0.266-2.344 \square$ & & {$[0.248-2.233 \square$} & \\
\hline \multirow[t]{2}{*}{ APO-B } & 0.563 & 0.548 & 0.539 & 0.521 & 0.917 & 0.885 & 0.719 & 0.602 \\
\hline & ¿0.086-3.672凸 & & ๑0.082-3.548₫ & & $\llbracket 0.282-2.980 \square$ & & ๔0.207-2.490凸 & \\
\hline \multirow[t]{2}{*}{ APO-E } & 0.988 & 0.473 & 0.987 & 0.467 & 1.009 & 0.373 & 1.007 & 0.497 \\
\hline & $\square 0.954-1.022 \square$ & & $\square 0.953-1.022 \square$ & & [0.989-1.029₫ & & {$[0.987-1.027 \square$} & \\
\hline
\end{tabular}

Table 4. Odds ratio (OR) (with 95\% CI) of Apo-A1 and

Lp(a) by tumor size in young male group ( $<55$ years old).

\begin{tabular}{cccccc}
\hline & Crude & & & Adjusted & \\
\cline { 5 - 6 } \cline { 5 - 6 } Apo-A1 & OR (95\% CI) & $P$ value & & OR (95\% CI) & $P$ value \\
\hline & 0.047 & 0.01 & & 0.051 & 0.01 \\
& $(0.005-0.485)$ & & & $(0.005-0.497)$ & \\
\hline
\end{tabular}


Table 5. Odds ratios (OR) (with 95\% CI) of

levels of 7 serum lipid by LNM in male and

female group respectively.

\begin{tabular}{|c|c|c|c|c|c|c|c|c|}
\hline & \multicolumn{3}{|c|}{ Male } & & \multicolumn{3}{|c|}{ Female } & \multirow[b]{3}{*}{$P$ value } \\
\hline & \multicolumn{2}{|l|}{ Crude } & \multicolumn{2}{|l|}{ Adjusted } & \multicolumn{2}{|l|}{ Crude } & Adjusted & \\
\hline & $\begin{array}{l}\text { OR } \\
(95 \% \\
\text { CI) }\end{array}$ & $P$ value & $\begin{array}{l}\text { OR }(95 \% \\
\text { CI })\end{array}$ & $P$ value & $\begin{array}{l}\text { OR } \\
\text { (95\% } \\
\text { CI) }\end{array}$ & $P$ value & $\begin{array}{l}\mathrm{OR}(95 \% \\
\mathrm{CI})\end{array}$ & \\
\hline \multirow[t]{2}{*}{ CHOL } & 0.93 & 0.633 & 0.928 & 0.628 & 0.852 & 0.119 & 0.925 & 0.484 \\
\hline & $\begin{array}{l}\square 0.691- \\
1.252 \square\end{array}$ & & $\begin{array}{l}\square 0.687- \\
1.254 \square\end{array}$ & & $\begin{array}{c}\square 0.696- \\
1.042 \square\end{array}$ & & $\begin{array}{l}\square 0.744- \\
1.150 \square\end{array}$ & \\
\hline \multirow[t]{2}{*}{ TG } & 0.988 & 0.955 & 0.991 & 0.967 & 0.798 & 0.082 & 0.873 & 0.306 \\
\hline & $\begin{array}{l}\square 0.645- \\
1.512 \square\end{array}$ & & $\begin{array}{l}\square 0.646- \\
1.5210\end{array}$ & & [0.619-1 & & $\begin{array}{l}\square 0.674- \\
1.132 \square\end{array}$ & \\
\hline \multirow[t]{2}{*}{ HDL-C } & 2.177 & 0.234 & 2.281 & 0.216 & 0.868 & 0.679 & 0.874 & 0.702 \\
\hline & $\begin{array}{l}00.604- \\
7.846 \square\end{array}$ & & $\begin{array}{l}\square 0.617- \\
8.433 \square\end{array}$ & & {$[0.444-1$} & & $\begin{array}{l}\square 0.439- \\
1.741 \square\end{array}$ & \\
\hline \multirow[t]{2}{*}{ LDL-C } & 0.85 & 0.431 & 0.851 & 0.44 & 0.823 & 0.167 & 0.924 & 0.608 \\
\hline & $\begin{array}{l}\square 0.568- \\
1.273 \square\end{array}$ & & $\begin{array}{l}\square 0.565- \\
1.281 \square\end{array}$ & & ¿0.625-1 & & $\begin{array}{l}\square 0.685- \\
1.248 \square\end{array}$ & \\
\hline \multirow[t]{2}{*}{ APO-A1 } & 2.339 & 0.293 & 2.721 & 0.232 & 0.412 & 0.061 & 0.471 & 0.121 \\
\hline & $\begin{array}{l}\square 0.481- \\
11.377 \square\end{array}$ & & $\begin{array}{l}\square 0.526- \\
14.72 \square\end{array}$ & & $\llbracket 0.163-1$ & & $\begin{array}{l}\square 0.182- \\
1.219 \square\end{array}$ & \\
\hline \multirow[t]{2}{*}{ APO-B } & 0.499 & 0.381 & 0.501 & 0.387 & 0.584 & 0.297 & 0.964 & 0.948 \\
\hline & $\begin{array}{l}\square 0.105- \\
2.360 \square\end{array}$ & & $\begin{array}{l}\square 0.104- \\
2.401 \square\end{array}$ & & {$[0.212-1$} & & $\begin{array}{l}\square 0.318- \\
2.926 \square\end{array}$ & \\
\hline \multirow[t]{2}{*}{ APO-E } & 1.016 & 0.352 & 1.017 & 0.326 & 0.992 & 0.393 & 0.999 & 0.89 \\
\hline & $\begin{array}{l}\square 0.983- \\
1.050 \square\end{array}$ & & $\begin{array}{l}\square 0.983- \\
1.051 \square\end{array}$ & & $\begin{array}{l}\square 0.974- \\
1.010 \square\end{array}$ & & $\begin{array}{l}\square 0.980- \\
1.018 \square\end{array}$ & \\
\hline
\end{tabular}

\section{Figures}

Thyroid cancer patients $(n=1586)$

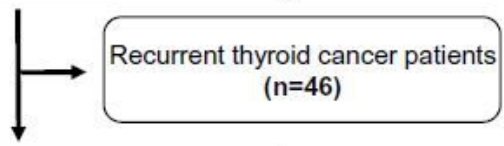

Primary thyroid cancer $(n=1540)$

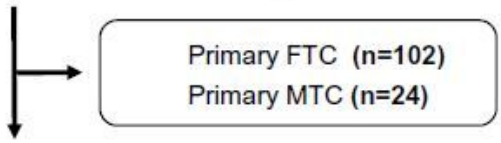

Primary PTC patients $(n=1414)$

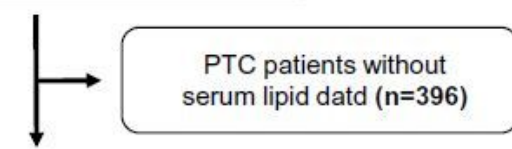

Primary PTC patients with serum lipid

examination before surgery $(n=1018)$ 
Figure 1

Flow diagram of the patient selection process.

Page 10/10 